\title{
The analysis of the power factor of the capacitive pulse load in the aviation variable frequency power supply system
}

\author{
Mengyu $\mathrm{He}^{1, \mathrm{a}}$, Yuanjun Zhou ${ }^{1, \mathrm{~b}}$ \\ ${ }^{1}$ School of Automation Science and electrical engineering, Beihang University, Beijing 100191, \\ China; \\ a297203259@qq.com, bzhouyuanjun@buaa.edu.cn
}

Keywords: variable frequency, impedance characteristics, nonlinear.

\begin{abstract}
More electric/all electrical aircraft" become the development trend in modern aviation, which leads a large increase of nonlinear devices. A capacitive load is formed when uses a capacitor filter to improve the quality of DC power supply. Coupled with the using of $360 \sim 800 \mathrm{~Hz}$ power supply system, the impedance characteristics of the system will become complex. This paper consider the general situation, deduces the parameter calculation expressions of the equivalent exchange RC series-parallel load. Then, combined with the example, the impact of of the impedance characteristics for changing frequency is analysised under aviation VF system (360 800Hz).
\end{abstract}

\section{Introduction}

"More electric/all electrical aircraft" become the development trend in modern aviation[1,2], which uses electric energy for replace most or all the power source of the aircraft (electricity, liquid, gas). This leads a large increase of the motor and power electronic devices in the aircraft power supply system. Thus, this kind of equipment tends to show nonlinear load characteristic for power grid, and leads to power waveform distortion, damages the power quality of the power grid. Capacitor filter is one of common methods to improve the quality of DC power supply for the power supply system, meanwhile, the capacitive load of the power grid is formed.

On the other hand, the innovation of power supply system in aircraft performance in the system of power supply: $360 \sim 800 \mathrm{~Hz}$ large capacity variable frequency AC power system is using gradually instead of the constant frequency of $400 \mathrm{~Hz}$ power supply on most of the aircraft[3,4]. The impedance characteristics of the load under Aviation VF power supply system $(360 \sim 800 \mathrm{~Hz})$ is different from that under the system of constant frequency $(400 \mathrm{~Hz})$ for the changing frequency of power supply.

This paper consider the general situation, deduces the parameter calculation expressions of the equivalent exchange RC series-parallel load. Then, combined with the example, the impact of of the impedance characteristics for changing frequency is analysised under aviation VF system (360 800Hz).

\section{Capacitive load of Aircraft AC power supply system}

\subsection{AC filter problem of AC/DC converter}

Auto Transformer Rectifier Units (ATRU) is used as the AC/DC converter in Power electronic load of aircraft power supply system, which is shown in Fig. 1. This converter alters 115V AC into $270 \mathrm{~V}$ DC and powers for different electronic load. In order to guarantee the power quality of the AC power grid, ATRU is required to adopt the structure of multi pulse such as 12 pulse ATRU, 18 pulse ATRU and 24 pulse ATRU. Fig. 1 illustrates the 18 pulse ATRU. 


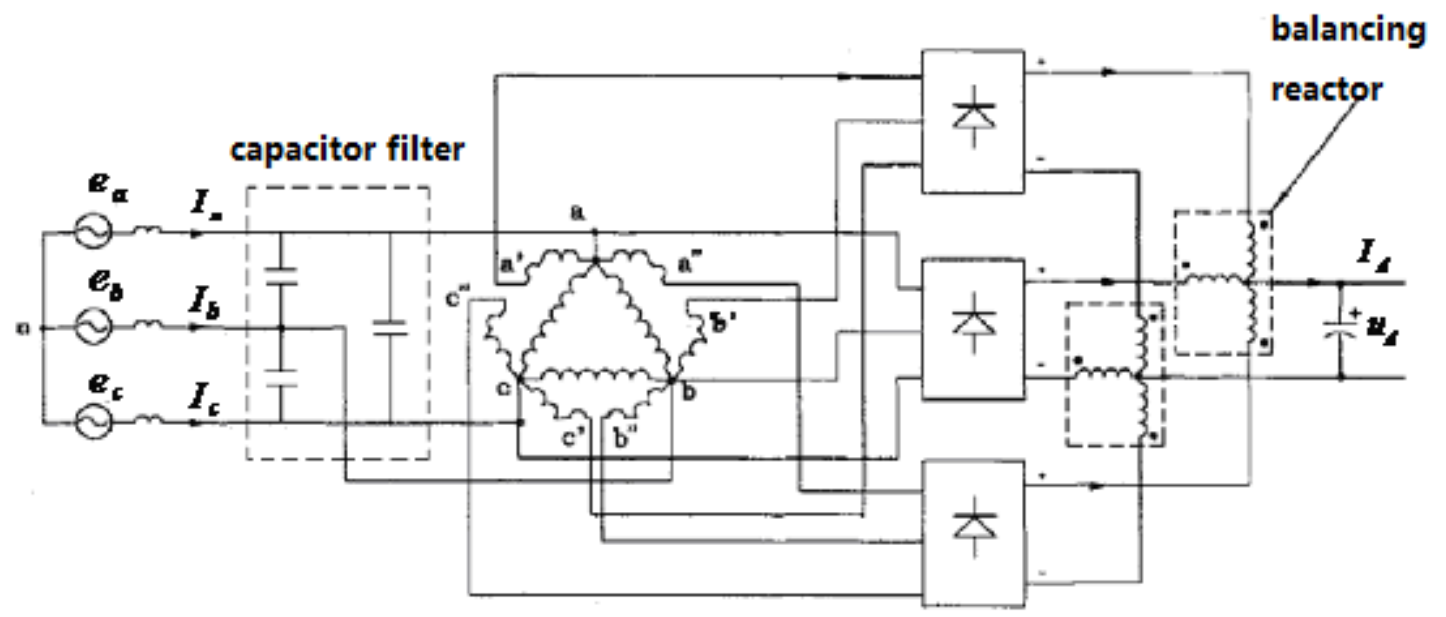

Fig. 1 the structure of 18 pulse ATRU

When the DC side is connected with the switching pulse load, even the multi pulse structure can not ensure the load characteristics. For example, voltage and current waveform of AC side is shown in Fig. 2 (a), while using 18 pulse ATRU as AC/DC converter and DC side connecting $1 \mathrm{kHz}$ pulse load. Obviously, the current waveform appears distortion. In this case, a capacitor filter in Fig. 1 is added in the AC input side in order to improve the effect of load to the power grid. The result is shown in Fig. 2 (b) that the current waveform is improved significantly.

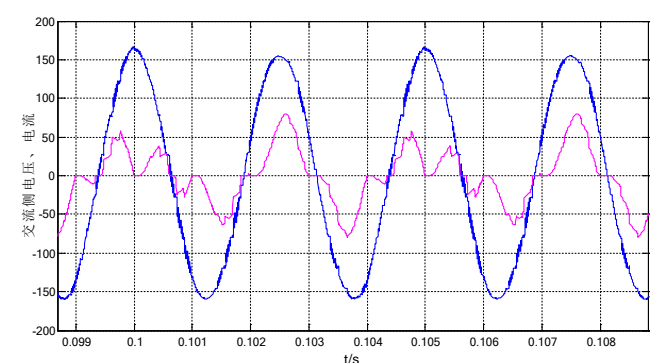

(a) the waveform before increasing

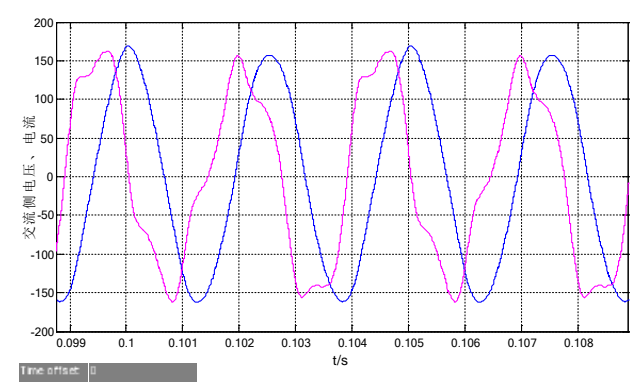

(b) the waveform after increasing

Fig. 2 the comparison charts before and after increasing

Due to the addition of the capacitor filter, the whole equipment turns into a capacitive characteristic load to the power grid.

\subsection{Simplified model of power supply system with capacitive load}

A simplified model of a single phase power supply circuit with capacitive load is shown in Fig. 3 (a). The ATRU and DC load are equivalent to be a load resistance $R_{L P}$, which is parallel with a filter capacitor $C_{P}$ in the model. $e_{a}$ is the back EMF of generator's A phase, and internal resistance, inductance are described as $r_{a}, L_{a}$. In order to analyze the power factor of the circuit, the structure of the load is transformed from partial to serial which is illustrated in Fig. 3(b). The parallel load $R_{L P}, C_{P}$ is equivalent to series load $R_{L S}, C_{S}$.

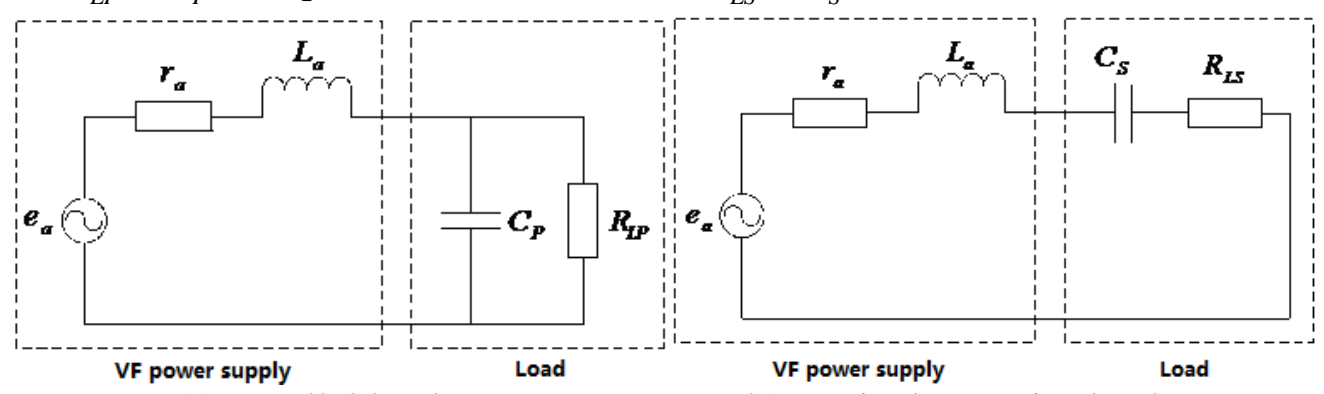

(a) Parallel load

(b) Equivalent series load

Fig. 3 Simplified model of power supply system with capacitive load

The impedance of parallel and series load will be: 


$$
\begin{aligned}
& R_{L S}=\frac{R_{L P}}{\left(\omega C_{P} R_{L P}\right)^{2}+1} \\
& X_{C S}=\frac{\omega C_{P} R_{L P}{ }^{2}}{\left(\omega C_{P} R_{L P}\right)^{2}+1}
\end{aligned}
$$

The power factor of the load is:

$$
P F=\frac{1}{\sqrt{1+\left(\omega C_{P} R_{L P}\right)^{2}}}
$$

By the formula $(1,2)$, the impedance and power factor of the load are determined by the effect of the phase load resistance $R_{L P}$ and the phase filter capacitance $C_{P}$, and is the function of the power supply electric angular velocity $\omega$. Besides, they are nonlinear.

\section{Impedance characteristics of capacitive load in variable frequency power supply}

The load impedance and power factor as shown in Fig. 3 will change for the altering angular velocity $\omega(\omega=2 \pi f)$ in the use of the aeronautic VF (360 800Hz) power supply.

\subsection{The effect of power frequency on load impedance}

By the formula (1), if $R_{L P}$ and $C_{P}$ are fixed, the changes of power frequency will lead to the changes of $X_{C P}$, and then trigger $R_{L S}$ and $X_{C S}$ changes. In this case, the formula (1) can be considered as a function of $\omega$.

Ask about the partial guide of $\omega$ on formula (1), there are:

$$
\begin{aligned}
\frac{d R_{L S}}{d \omega} & =\frac{-2 \omega C_{P}{ }^{2} R_{L P}{ }^{3}}{\left[\left(\omega C_{P} R_{L P}\right)^{2}+1\right]^{2}} \\
\frac{d X_{C S}}{d \omega} & =\frac{C_{P} R_{L P}{ }^{2}-\omega^{2} C_{P}{ }^{3} R_{L P}{ }^{4}}{\left[\left(\omega C_{P} R_{L P}\right)^{2}+1\right]^{2}}
\end{aligned}
$$

By the formula (3) we can know that when $\omega$ changes, $R_{L S}$ is monotonous decreasing, and $X_{C S}$ has extreme value. This extreme value will arise when meet $\omega C_{P} R_{L P}=1$, and $\frac{d X_{C S}}{d \omega}=0$. At this point, the maximum capacitive is shown as a fixed value $X_{C S}=\frac{1}{2} R_{L P}$. Meanwhile, $R_{L S}=\frac{1}{2} R_{L P}$, and the power factor is $\frac{1}{\sqrt{2}}=0.707$.

For example, the power supply phase voltage is $115 \mathrm{~V}$, the ATRU transform load power is $6 \mathrm{~kW}$, and the phase filter capacitor is $60 \mu \mathrm{F}$. The power of each phase is $2 \mathrm{~kW}$ when ignoring the power loss, and the equivalent phase load resistance $R_{L P}$ is $6.6 \Omega$. Thus, $X_{C S}$ appear maximum value when the power frequency is $400 \mathrm{~Hz}$ and $\omega=2525 \mathrm{rad} / \mathrm{s}$. Simulation results of different frequencies are shown in Fig. 4. 


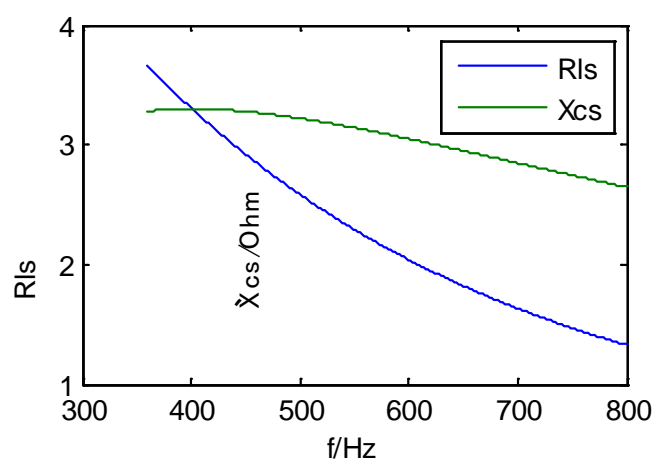

(a) the process of $R_{L S}$ and $X_{C S}$ change

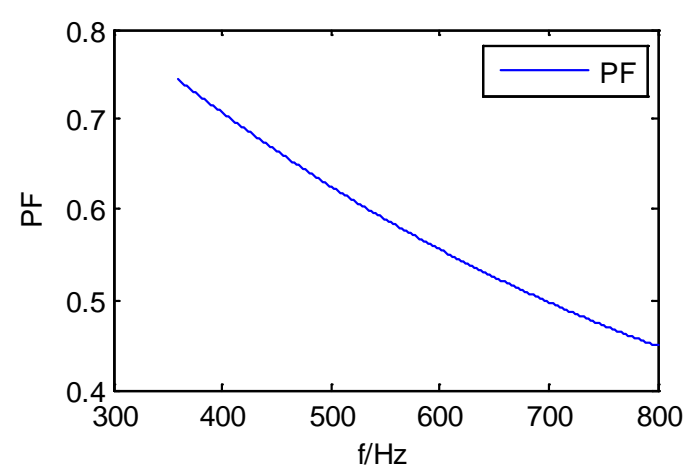

(b) the process of $P F$ change

Fig. 4 Impedance characteristic change of VF power supply $\left(R_{L S}, X_{C S}, P F\right)$

\subsection{Influence of load power varying}

The variation of impedance and power factor is nonlinear when output DC power of ATRU changes. Three types of power are chosen to simulate: $6 \mathrm{Kw}\left(\mathrm{R}_{\mathrm{LP}}=6.6 \Omega\right), 9 \mathrm{~kW}\left(\mathrm{R}_{\mathrm{LP}}=4.4 \Omega\right)$, $4.5 \mathrm{Kw}\left(\mathrm{R}_{\mathrm{LP}}=8.82 \Omega\right)$. The phase filter capacitor is $60 \mu \mathrm{F}$. When $f$ changes, the changing curve of $R_{L S}$ and $X_{C S}$ corresponding to different power load can be drawn according to the formula (1), which is shown in Fig. 5.

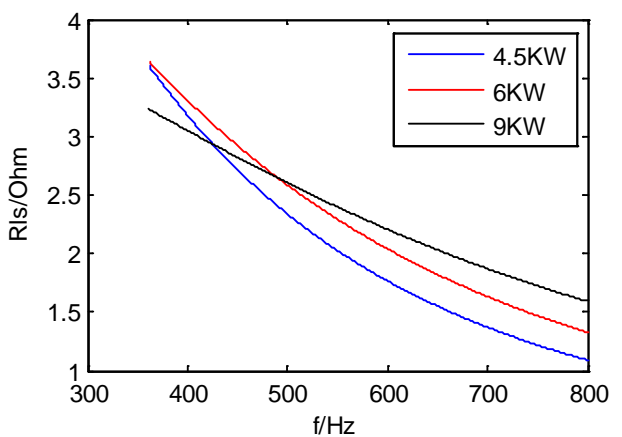

(a) the changing curve of $R_{L S}$

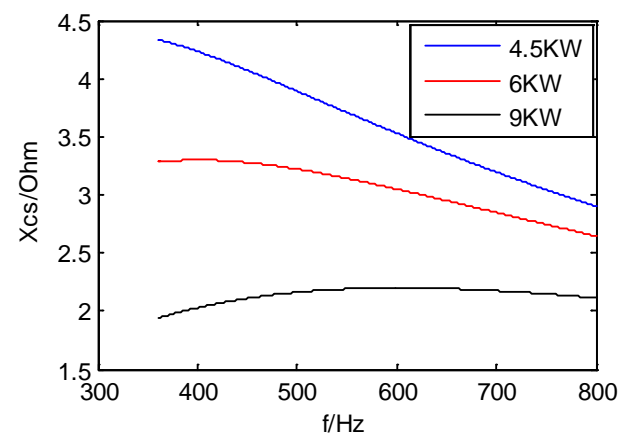

(b) the changing curve of $X_{C S}$

Fig. 5 the changing curve of $R_{L S}$ and $X_{C S}$ with $f$ varying

\subsection{Influence of filter parameters}

The variation of impedance and power factor is also nonlinear when filter parameters of ATRU changes. Three kinds parameter of filter are chosen to simulate: $C_{P}=30 \mu \mathrm{F}, C_{P}=60 \mu \mathrm{F}$, and $C_{P}=90 \mu \mathrm{F}$. The output DC power of ATRU is 9KW. When $f$ changes, the changing curve of $R_{L S}$ and $X_{C S}$ corresponding to different filter parameter can be drawn according to the formula (1), which is shown in Fig. 6.

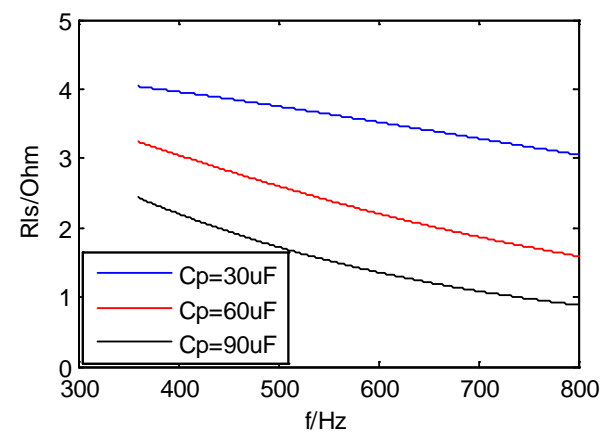

(a) the changing curve of $R_{L S}$

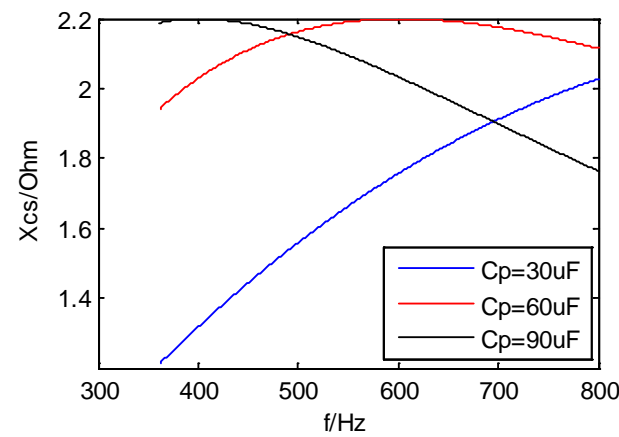

(b) the changing curve of $X_{C S}$

Fig. 6 the changing curve of $R_{L S}$ and $X_{C S}$ with $f$ varying 


\section{Conclusion}

This paper consider the general situation, deduces the parameter calculation expressions of the equivalent exchange RC series-parallel load in simplified model of aviation power system. Based on this, the impedance characteristics of the capacitive load under the aviation VF system $(360 \sim 800 \mathrm{~Hz})$ are analyzed, and the effects of power frequency, load power and filter parameters to the impedance characteristics of capacitive load are pointed out.

\section{References}

[1] Chang J., Wang A.. New VF-Power System Architecture and Evaluation for Future Aircraft, J. IEEE Trans. Aerospace and Electronic Systems. 2006, 42(2): 527-539.

[2] Rosero J A, Ortega J A, Aldabas E, etal. Moving Towards a More Electric Aircraft, J. IEEE Aerospace and Electronic Systems Magazine, 2007, 22(3): 3-9.

[3] Emadi K, and Ehsani M. Aircraft power systems: technology, state of the art, and future trends, J. IEEE A\&E Systems Magazine, 2000, 15(1): 28-32.

[4] Cheng G. The current situation and development of large civil aircraft power system, J. Civil Aircraft Design and Research, 2008, 4(1): 1-5. 